*Generalized Linear Mixed Models.

GENLINMIXED

/DATA_STRUCTURE SUBJECTS=Horse*Session REPEATED_MEASURES=Trial COVARIAN $\bar{N} E$ TYPE$=$ DIAGONAL

/FIELDS TARGET=Outcome TRIALS=NONE OFFSET=NONE

/TARGET OPTIONS DISTRIBUTION=BINOMIAL LINK=PROBIT

/FIXED EFFECTS=Session Horse picture Other picture

USE INTERCEPT=TRUE

/RANDOM USE INTERCEPT=TRUE SUBJECTS=Horse

COVARIANCE TY $\bar{P} E=V A R I A N C E$ COMPONENTS SOLUTION=FALSE

/BUILD O $\bar{P} T I O N S$ TARGET $\bar{C} A T E G O R Y$ ORDER=ASCENDING

INPUTS CĀTEGORY ORDER=A $\bar{S} C E N D I N G \quad \bar{M} A X$ ITERATIONS=100 CONFIDENCE LEVEL=95

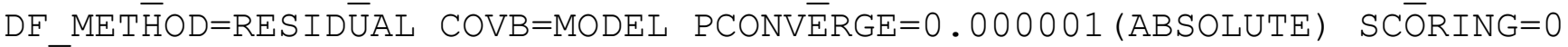

SIN $\bar{N} G U L A R=0.000000000001$

/EMMEANS TABLES=Session COMPARE=Session CONTRAST=PAIRWISE

/EMMEANS TABLES=Horse picture COMPARE=Horse picture CONTRAST $=$ PAIRWISE

/EMMEANS TABLES=Other picture COMPARE=Other picture CONTRAST $=$ PAIRWISE

/EMMEANS_OPTIONS SCALE=ORIGINAL PADJUST=SEQSIDAK.

\title{
Generalized Linear Mixed Models
}

\begin{tabular}{lll}
\multicolumn{2}{l}{ Notes } \\
Output Created & \multicolumn{1}{c}{ 08-NOV-2020 12:30:58 } \\
\hline Comments & & \\
\hline Input & Active Dataset & DataSet1 \\
\cline { 2 - 3 } & Filter & <none $>$ \\
\cline { 2 - 3 } & Weight & $<$ none $>$ \\
\cline { 2 - 3 } & Split File & $<$ none $>$ \\
\cline { 2 - 3 } & N of Rows in Working Data & \\
& File & \\
&
\end{tabular}

Case Processing Summary

\begin{tabular}{lr|r} 
& $\mathrm{N}$ & Percent \\
\hline Included & 270 & $100,0 \%$ \\
\hline Excluded & 0 & $0,0 \%$ \\
\hline Total & 270 & $100,0 \%$ \\
\hline
\end{tabular}

\section{Model Summary}

\begin{tabular}{llr}
\hline Target & Outcome $+/-$ \\
\hline Probability Distribution & & Binomial \\
\hline Link Function & Probit & \\
\hline Information Criterion & Akaike Corrected & \\
\cline { 2 - 3 } & Bayesian & 994,130 \\
\hline
\end{tabular}


Information criteria are based on the -2 log likelihood $(970,997)$ and are used to compare models. Models with smaller information criterion values fit better.

\title{
Data Structure ${ }^{a}$
}

\begin{tabular}{lrr|rr|r} 
& \multicolumn{2}{c}{$\begin{array}{c}\text { Subjects } \\
\text { Session }\end{array}$} & $\begin{array}{c}\text { Repeated } \\
\text { Measures } \\
\text { Trial }\end{array}$ & $\begin{array}{c}\text { Target } \\
\text { Outcome +/- }\end{array}$ \\
\hline Data for First Subject & 1 & 1 & 1 & 0 \\
\cline { 2 - 6 } & 1 & 1 & 2 & 1 \\
\hline & 1 & 1 & 3 & 0 \\
\hline & 1 & 1 & 4 & 1 \\
\hline & 1 & 1 & 5 & 1 \\
\hline & 1 & 1 & 6 & 1 \\
\hline Total Number of Levels & 1 & 1 & 7 & 1 \\
\hline & 1 & 1 & 8 & 0 \\
\hline
\end{tabular}

a. Target: Outcome +/-

\section{Classification}

\section{Overall Percent Correct $=75,9 \%^{a}$}

\author{
Predicted
}

\begin{tabular}{llr|r}
\multicolumn{2}{l}{ Observed } & 0 & \multicolumn{1}{c|}{1} \\
\hline 0 & Count & 12 & 54 \\
\cline { 2 - 4 } & $\%$ within Observed & $18,2 \%$ & $81,8 \%$ \\
\hline 1 & Count & 11 & 193 \\
\cline { 2 - 4 } & $\%$ within Observed & $5,4 \%$ & $94,6 \%$ \\
\hline
\end{tabular}

a. Target: Outcome +/-

\section{Fixed Effects ${ }^{a}$}

\begin{tabular}{lr|r|r|r} 
Source & F & df1 & df2 & \multicolumn{1}{c}{ Sig. } \\
\hline Corrected Model & 1,378 & 24 & 245 &, 117 \\
\hline Session & 2,517 & 6 & 245 &, 022 \\
\hline Horsepicture & 1,465 & 9 & 245 &, 162 \\
\hline Otherpicture1 &, 784 & 9 & 245 &, 631 \\
\hline
\end{tabular}

Probability distribution: Binomial Link function: Probit ${ }^{a}$

a. Target: Outcome +/- 
Fixed Effects

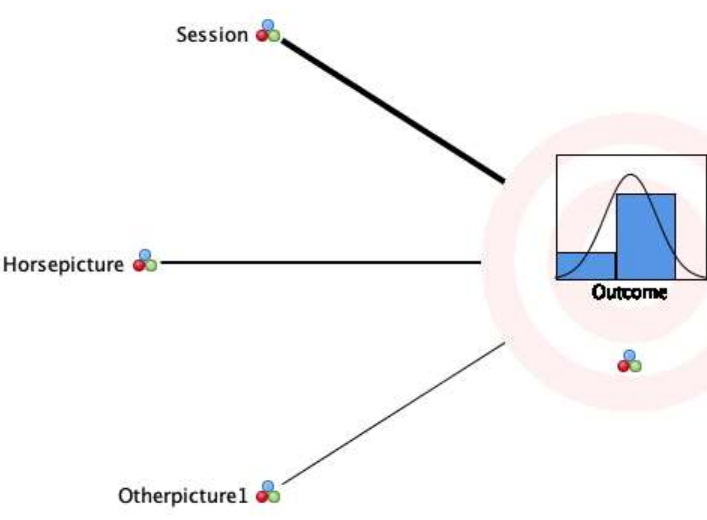

\section{Overall Test Results}

\begin{tabular}{r|rrrr}
\multicolumn{1}{l}{$\mathrm{F}$} & $\mathrm{df1}$ & \multicolumn{1}{c}{ df2 } & Sig. \\
\hline, 552 & & 9 & 245 &, 836 \\
\hline
\end{tabular}

